\title{
Automatic Ambulance Rescue With Intelligent Traffic Light
} System

\author{
K.Sangeetha ${ }^{1}$, P.Archana ${ }^{2}$, M.Ramya ${ }^{3}$, P.Ramya ${ }^{4}$, \\ M.E, B.E, B.E,B.E \\ ${ }^{I}$ Assistant Professor, ECE,SNS CollegeOf Engineering, Coimbatore \\ ${ }^{2}$ Final year student, ECE,SNS College Of Engineering,Coimbatore \\ ${ }^{3}$ Final year student, ECE,SNS College Of Engineering,Coimbatore \\ ${ }^{4}$ Final year student, ECE,SNS College Of Engineering, Coimbatore
}

\begin{abstract}
The road accident in the present era is increased to greater extent. The loss of human life due to accident must be avoided. Traffic congestion and tidal flow are major facts that cause delay to ambulance. In order to save human life from accidents we introduce a scheme called ITLS (Intelligent Traffic Light system).The main concept behind this scheme is to provide a smooth flow for the emergency vehicles like ambulance to reach the hospitals in time and thus minimizing the delay caused by traffic congestion. The idea behind this scheme is to implement ITLS which would control automatically the traffic lights in the path of the ambulance. With the help of thisIntelligent Transportation System integrated with theGPS the current scenario of trafficcongestion can be solved to an extent. This scheme is fully automated controls the traffic lights, helping to reach the hospital in time. Here we track the ambulance location using GPS units, and its sends the data to the traffic lights through embedded system .This system control the traffic lights and save the time in emergency periods.
\end{abstract}

Keywords: - ITLS,GPS.

\section{INTRODUCTION}

Emergency occurs anywhere at any location, at any time, and in various ways will make one at risk. These situations require a speedy response. So it is very crucial and important to establish direct, fast and efficient technique without delay. With the increasing number of population in the metropolitan areas already existing problem of poor traffic congestion has grown to an alarming event. This problem has to be properly analyzed and the appropriate measures have to be taken. Often rural areas are devoid of the traffic congestion. The proper care is to be given to the urban areas mainly focusing on to the metropolitan cities. Even if each and every vehicle passing through the traffic has its own need, the prior importance is given to the Ambulance and other emergency vehicles which needs to wait longer time on the traffic thereby increasing the probability of risk .Transportation of a patient to hospital in emergency seems quite simple but in actual it is pretty difficult during peak hours. Optimum utilization of the time after an accident is actually the golden hours as a measure of effectiveness of an emergency response service provider system. Recovery action should be taken immediately. Congestion can be solved to an extent. In addition to this, in the case of givingprior importance to the ambulance and otheremergency vehicles, their chances of misusing thevehicles by some people for their own benefit is veryhigh. The older technology uses RF transmitter is mounted on atop of the ambulance and RF receivers are placed in every road leading to the signal at a suitable distance from the traffic signal. Initially the driver of the ambulance switches on the transmitter through a switch placed on the steering wheel.This makes the receiver output to go high and thereby interrupting the microcontroller .At the beginning of the interrupt sub routine, all the port pins are scanned to determine in which lane the ambulance is approaching and the corresponding lane is made green. In order to tackle these problems, this paper hascome up with a proposed system using the advancedGPRS Technologies for faster data transmission. The ambulance is controlled by the control unit and also controls the traffic light according to the ambulance location and thus reaching the hospital safely.

\section{IMPLEMENTATION}

The system uses two microcontrollers and the GPS installed in Ambulance identifies the latitude and longitude of the particular place thereby finding the location of the ambulance unit. GPRS 3G Modem installed in the ambulance and the traffic junction helps to communicate with each other at a greaterspeed and at greater coverage. Initially, the mode is set the by using MODE switch. MODE switch is used to indicate whether the situation is critical ornot. The Latitude and longitude position of the ambulance was obtained by the GPS. The microcontroller receives the GPS value. After receiving the controller compares the GPS value in PC via RS232 in control room. If the GPS value is nearby indicating that the ambulance or the emergency vehicle is 
near to the traffic junction, then the corresponding signal in traffic is green for the Ambulance to pass through without waiting. Alarm is ON for indicating that the Ambulance is in a nearby location. In order for the GPS to work, a network ofsatellites was placed into an orbit around the planetEarth, each broadcasting a specific signal. This signalcan be achieved by a low technology aerial.LCD Display is used for displaying the status of the processing. An LCD consists of two glass panels, with the liquid crystal material sandwitched in between them.LCD doesn't generate light and so light is needed to read the display.Driver circuit is an electrical circuit used to power the LCD.

This technique consists of two main units which co-ordinates with each other and makes sure that theAmbulance reaches the hospital without any delay.

The system consist of two unit

1) Ambulance unit.

2)Traffic junction unit .

\section{III . AMBULANCE UNIT}

The GPS installed in the ambulance unit senses the location of an ambulance.The controller calculates the direction of the ambulance using gps receiver and send it to the RF transmitter. And finally signal was sent to the traffic unit to make the signal green ensuring that the ambulance reaches the traffic without any time delay shown in figure.

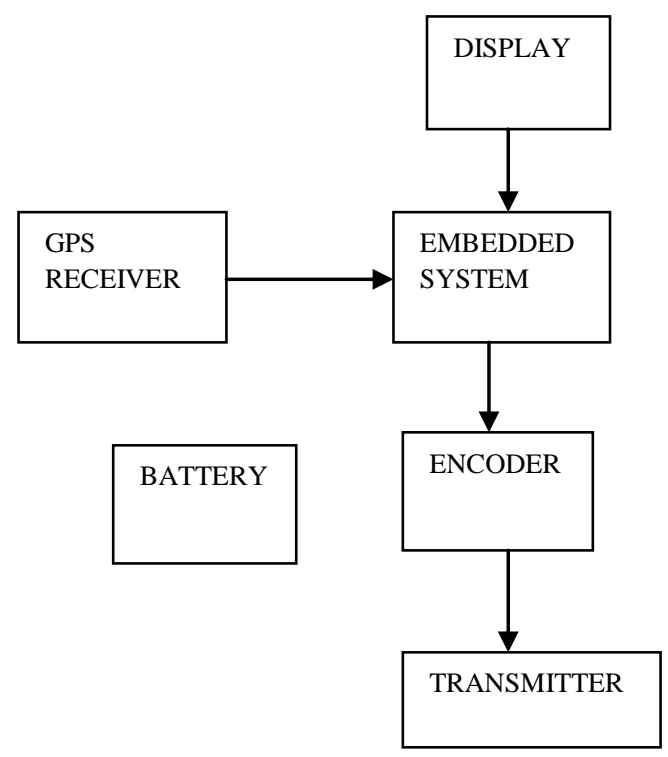

Fig .1 AMBULANCE UNIT

Ambulance unit consists of battery, GPS receiver, embedded system, encoder, transmitter keypad and display. In ambulance unit a GPS receiver is connected to the embedded system, It using the Global Positioning System to determine the location of ambulance, the determined location are sending directly to the embedded system, Embedded system is programmed to analyze the signals and it data transmits to the encoder devices. An encoder is a device, circuit, transducer, software program, algorithm or person that converts information from one format or code to another, this data transmitting to the receiver through transmitter. Embedded system is 32 bit PIC 16F887(Peripheral Interface Controller). The inputs are given through the pins 10-13. This data is encoded and can be collected at pin 17. It is given to the transmitter data input pin no 2, A0-A7 is the address pins and AD8-AD11 is the address and data pins using this signal collision is avoided. When the transmission enable signal is low data AD8-AD11 are acts as a data pins and give the data to data out. $\mathrm{V}_{\mathrm{DD}}$ is connected with the supply. When TE is get high the encoder stops the cycle and $\mathrm{D}_{\text {out }}$ is stopped.The supply is noise filtered by capacitors. Diode prevents encoder from wrong connection. Resistor across the osc1 and osc 2 supports oscillator.

\section{TRAFFIC JUNCTION UNIT}

Signal controller unit consists of receiver, decoder, embedded system, traffic signal. Signals from the ambulance unit received by the signal controller unit.Here signals are sending to the decoder, these devices doing reverse operation of an encoder undoing the encoding so that the original information can be retrieved. The same method used to encode is usually just reversed in order to decode, then decoded signal transmitting to 
the embedded system, embedded system is programmed to control the traffic signals. Decoder is a device, circuit, transducer, software program, algorithm or person that converts information from one format or code to another, the decoded data transmitted Embedded system. In this circuit HT12D decoder IC is used to decode the data. The input is given to the pin 14 from the receiver. This data is decoded and can be collected at pin 10-13. A0-A7 is the address pin using this signal collision is avoided. $V_{D D}$ is connected with the supply. The supply is noise filtered by capacitors. Diode prevents encoder from wrong connection. Resistor across the osc1 and osc2 supports oscillator.Embedded system used is PIC 16f887.Traffic junction unit is shown in the fig 2.

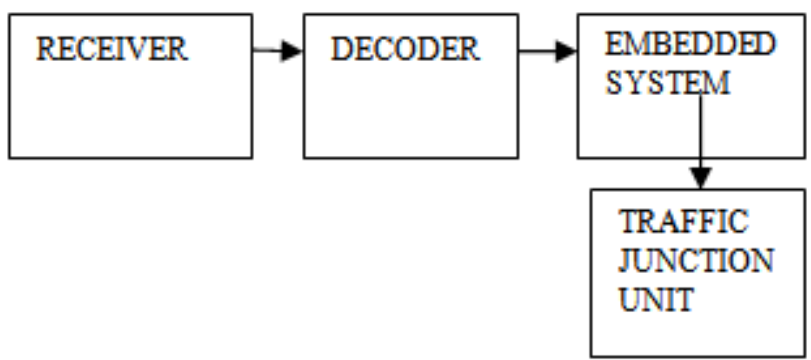

Fig. 2 TRAFFIC JUNCTION UNIT

IV.

NODE ACCESS AND CONTROL

The nodes in the ambulance path are accessed and controlled only when the ambulancereaches adistance of around say $100 \mathrm{~m}$ from the node. GPS provide location and direction of the ambulance. Consider a traffic junction unit has four lane .Each lane was in direction such as east,west,north, south. According to the direction of the ambulance the corresponding lane will be turned ON. Gps was fixed on the ambulance if it is from the east side. Gps receiver obtain the direction of the ambulance provides the information to traffic unit and the corresponding lane will be turned green and immediately the remaining lane will be turned to red.

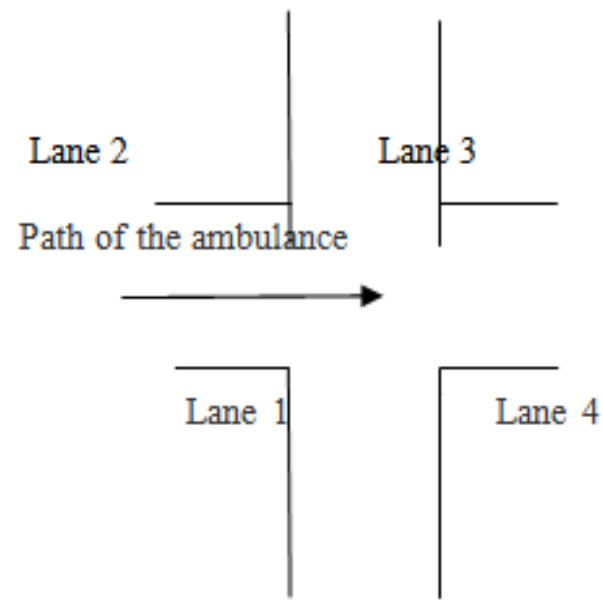

V. RESULTS

The model of our ITS is developed partially to some ex-tent. Considering the cost and time constraints the ambulance unit and the traffic junction (node section) have been developed and also we are in process of linking together the ambulance unit and the server. But for the pro-totype model we have linked the ambulance unit and the traffic junction using a RF transmitter.

The Figure 4 shows the ambulance unit consisting of GPS module which tracks the current position of the ambulance all the time. The unit has a controller which stores the actual coordinates of the locations $(1 \mathrm{~km}$ marking and the traffic signal node). When the GPS coordinates matches with that of the stored coordinates, it is said to have reached that place and then a signal is sent to the traffic junction (shown in Figure 3) using RF transmitter which works at $433 \mathrm{MHz}$. The GPS receiver have an resolution of $3 \mathrm{~m}$ and transmits the data serially at a baud rate of $9600 \mathrm{bps}$. The LCD HD44780 is installed in this unit to continuously display the positional values of the ambulance. The traffic junction has RF receiver which receives the signal (START signal) which makes the junction to work in ambulance mode. When the ambulance crosses the junction it again comes back to the normal mode. The Table1 shows the range of GPS values of $1 \mathrm{~km}$ marking and the traffic signal node which is stored in the ambulance unit's controller and compared with its location continuously. 
Table1.Showing the GPS coordinates of stored and actual values Figure 3. The Traffic Junction Unit .The Figure 3 shows the traffic signal junction with the three way lane model. This unit consists of a RF receiver which also works at $433 \mathrm{MHz}$. The junction operates as per the data transmitted by the ambulance unit. The data received by the RF receiver is transmitted to the controller at the rate of $3 \mathrm{kbps}$. When the data received is 0 , it operates in the normal mode. When 1 is received, the traffic signal shifts to ambulance mode and the particular direction is made green. When 2 is received, it return backs to normal mode.

The Figure 3 shows the GPS module showing the coordinates. The GPS value calculated will be in NMEA standard with numerous statements. A particular statement is selected, here we took GPGGA and the longitude and latitude values are retrieved from it and displayed in the LCD by the controller.

Table1.Showing the GPS coordinates of stored and actual values

\begin{tabular}{|l|l|l|l|}
\hline $\begin{array}{l}\text { LATITUDE } \\
\text { (STORED) }\end{array}$ & $\begin{array}{l}\text { LONGITUD } \\
\text { E(STORED) }\end{array}$ & $\begin{array}{l}\text { LAT } \\
\text { (ACTUAL) }\end{array}$ & $\begin{array}{l}\text { LONG } \\
\text { (ACTUAL) }\end{array}$ \\
\hline $\begin{array}{l}7937.3400- \\
7937.3450 \\
(\text { START })\end{array}$ & $\begin{array}{l}07959.4403 \\
\text { (START) }\end{array}$ & 7936.3424 & 07959.4403 \\
\hline
\end{tabular}

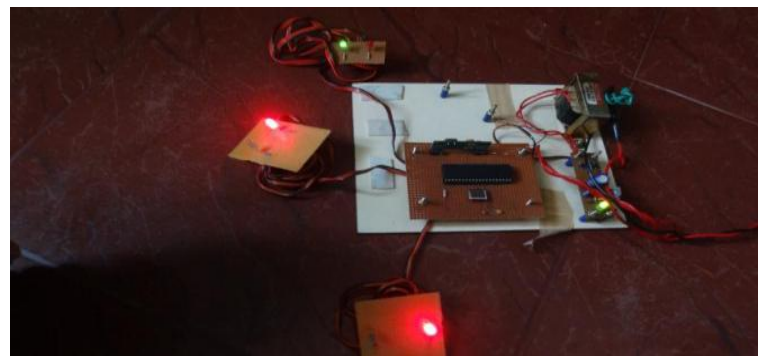

Fig. 3 TRAFFIC JUNCTION UNIT

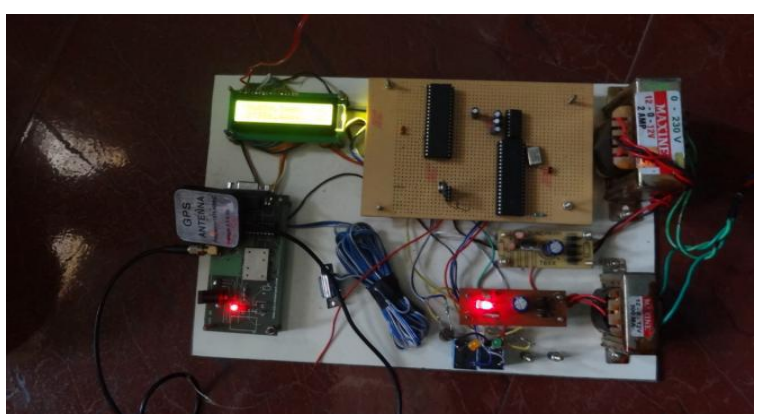

Fig. 4 AMBULANCE UNIT

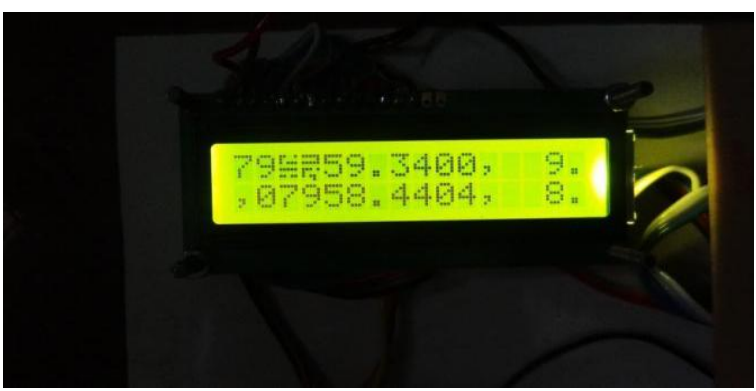

Fig. 5 GPS POSITION IN LCD

\section{CONCLUSION}

In this paper, a novel idea is proposed for controlling the traffic signals in favor of ambulances during the accidents. With this system the ambulance can be reached to the hospital without time lag. The ITLS can be proved to be effectual to control not only ambulance but also authoritative vehicles. Thus ITLS if implemented 
in countries with large population like INDIA can produce better results. The ITLS is more accurate with no loss of time. If two ambulance reaches the lane opposite to each other at the same time ,priority is given based on FIFO. The probability of the ambulance reaches the lane opposite to each other at the same time is less.

\section{REFERENCES}

[1] Wang wei, fan hanbo, traffic accident Automatic detection and remote alarm Device

[2] Zhaosheng yang. Study on the schemes of Traffic signal timing for priority vehicles Based on navigation system, 2000.

[3] Xiaolin lu, develop web gis based Intelligent transportation application Systems with web service technology, Proceedings of internationalconference on its telecommunications, 2006.

[4] Katsunori tawara, naoto mukai, traffic Signal control by using traffic Congestion prediction based on Pheromone model, proceedings of 22nd International conference on tools with Artificial intelligence, 2010.

[5] Malik Tubaishat, Qi Qi, Yi Shang, Hongchi Shi "Wireless Sensor-Based Traffic Light Control" IEEE CCNC 2008 proceedings 1-4244-1457-1/08

[6] Qingfeng Huang and Ying Zhang. "Dynamic balancing of push and pull in a distributed traffic information system." In IEEE ConsumerCommunications and Networking Conference (CCNC 2007), 2007.

[7] Jianhou Gan, Lingyun Yuan, Zhongqi Sheng and Tianwei Xu, "Construction and Implementation of an Integrated WSID TrafficMonitoring Network System", Proc. 21st annual international conference on Chinese control and decision conference, 2009, pp. 4726-4731.

[8] Xu Li, Wei Shu, Minglu Li, Hong-Yu Huang, Pei-En Luo, Min-You Wu, "Performance Evaluation of Vehicle-Based Mobile SensorNetworks for Traffic Monitoring" IEEE transactions on vehicular technology, May 2009, vol. 58, no. 4, pp. 1647-1653.

[9] Ben-Jye Chang, Bo-Jhang Huang and Ying-Hsin Liang, "Wireless Sensor Network-based Adaptive Vehicle Navigation in Multihop-Relay WiMAX Networks", Proc. 22nd International Conference on Advanced Information Networking and Applications (AINA), 2008,pp. 56-63.

[10] Sensor node information available via www aten.wikipedia.org/wiki/Traffic_light_ control_and_coordination.

\section{BIOGRAPHY}

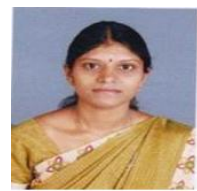

MS.K.SANGEETHA Assistant Professor Dept of ECE SNS COLLEGE OFENGINEERING Coimbatore.

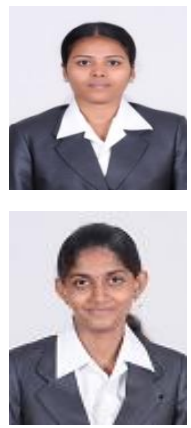

MS.P.ARCHANA currently pursing B.E(ECE) in SNS COLLEGE OFENGINEERING at Coimbatore.

MS.M.RAMYAcurrently pursing B.E(ECE) in SNS COLLEGE OFENGINEERING at Coimbatore.

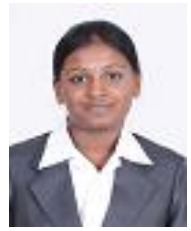

MS.P.RAMYA currently pursing B.E(ECE) in SNS COLLEGE OFENGINEERING at Coimbatore. 